\title{
EDITORIAL
}

\section{Cardiac hypertrophy and oxidative stress: a leap of faith or stark reality?}

\section{Lang}

Heart 2002;87:316-318

While the role of oxidative stress in diseases like hypercholesterolaemia, atherosclerosis, and diabetes is becoming increasingly well established, its involvement in the development of cardiac hypertrophy is more controversial

Correspondence to: Dr Derek Lang, Wales Heart Research Institute, University of Wales College of Medicine, Heath Park, Cardiff CF14 4XN, UK Langd@cf.ac.uk t is well known that cardiovascular diseases such as hypertension, valvular dysfunction, and myocardial infarction are associated with cardiac hypertrophy, which is itself an independent risk factor for sudden death. Less certain, however, is the identity of the molecular mechanisms involved in the hypertrophic process. The role of oxidative stress in the pathophysiology of diseases like hypercholesterolaemia, atherosclerosis, and diabetes is becoming increasingly well established. In contrast, the involvement of oxidative stress in the development of cardiac hypertrophy is more controversial, though with the evidence now available this concept does not require the great leap of faith once envisaged.

\section{OXIDATIVE STRESS IN FRIEDREICH'S ATAXIA?}

The term "oxidative stress" has become something of a buzz phrase in recent years, being implicated in countless disease processes. What is oxidative stress? It is described as a disturbance in the balance between the production of reactive oxygen species (ROS) and antioxidant defences in favour of the former. Examples of such ROS are superoxide anions $\left(\mathrm{O}_{2}^{-}\right)$, hydroxyl radicals, nitric oxide (NO) and peroxynitrite. Potential sources of these molecules include mitochondrial respiration, cytochrome p450s, xanthine oxidase, $\mathrm{NAD}(\mathrm{P}) \mathrm{H}$ oxidases, NO synthase, and others.

In the current issue of Heart Hausse and colleagues $^{1}$ ( $p$ 000) describe an interesting link between oxidative stress and the cardiac hypertrophy seen in the neurodegenerative disease Friedreich's ataxia (FRDA). In their study administration of the antioxidant idebenone, a short chain analogue of co-enzyme $\mathrm{Q}_{10}$, produced a significant reduction in cardiac hypertrophy in 50\% of the study cohort. A further striking link between oxidative stress and the pathology of FRDA is highlighted in another autosomal recessive disorder that results in a defect in the vitamin E transfer protein gene. Affected individuals have very low serum concentrations of this antioxidant vitamin and demonstrate the FRDA phenotype. ${ }^{2}$

\section{OXIDATIVE STRESS: THE SOURCE?}

These observations beg the question as to the source of the oxidative stress seen in FRDA. A possible answer is provided by various studies that now suggest dysfunctional mitochondria in the cardiac myocytes to be the source of the ROS. Given that cardiac myocyte mitochondria can generate ROS during heart failure, ${ }^{3}$ treatment with an antioxidant could provide significant benefit in preventing the transition from compensatory hypertrophy to cardiomyopathy and failure often seen in FRDA.

The causative mechanism that underlies the FRDA phenotype is a defect in the gene that encodes for a widely expressed protein frataxin, which is located in the mitochondrion and found in decreased amounts in FRDA. It has been suggested that decreased or absent frataxin impairs early antioxidant defences in FRDA. ${ }^{4}$ In the face of continuous oxidative damage to ironsulphur clusters within the mitochondria in the absence of superoxide dismutase, mitochondria become dysfunctional and as a consequence become overloaded with iron. This process could lead to an increased sensitivity to subsequent oxidative damage or increased susceptibility to cardiac myocyte apoptosis or necrosis. Exactly how decreased or absent frataxin results in defective antioxidant defences has not, however, been established with certainty.

Lodi and colleagues ${ }^{5}$ have recently suggested that a deficit in cardiac bioenergetics, as measured by cardiac phosphocreatine/ATP ratios, precedes the development of hypertrophy in FRDA patients and, furthermore, that mitochondrial dysfunction is the most likely cause of this deficiency. The precise nature of this abnormality remains unclear, however, though iron overload and decreased mitochondrial aconitase activity seem to be key features. It is therefore becoming apparent that in FRDA mitochondrial dysfunction precedes hypertrophy, which itself is the forerunner of cardiomyopathy and heart failure.

\section{OXIDATIVE STRESS IN OTHER CARDIOVASCULAR DISEASES?}

In other cardiovascular diseases also characterised by cardiac hypertrophy causative molecular mechanisms appear to be more firmly established than they are in FRDA. For instance, a recent review ${ }^{6}$ describes an array of intracellular signalling mechanisms that may be involved in both

Abbreviations: All, angiotensin II; $A C E$, angiotensin converting enzyme; FRDA, Friedreich's ataxia; NO, nitric oxide; ROS, reactive oxygen species 
cardiac hypertrophy and heart failure. Of particular interest are the pathways linked to the multifunctional agent angiotensin II (AII).

Raised plasma concentrations of AII are associated with many cardiovascular diseases and AII is capable of inducing cardiac hypertrophy and remodelling. Further evidence suggests that AII induces these growth effects via its ability to induce the overproduction of $\mathrm{O}_{2}^{-}$anions and the establishment of a pro-oxidising environment. Stimulation of AII type I receptors is thought to be linked to the upregulation of the $\mathrm{NAD}(\mathrm{P}) \mathrm{H}$ oxidase system, while $\mathrm{O}_{2}^{-}$anions may also be produced following activation of the small $\mathrm{G}$ protein Racl. We have previously shown that plasma concentrations of AII are raised in a pressure overload guinea pig model of left ventricular hypertrophy, ${ }^{7}$ and that this model is characterised by vascular oxidative stress and endothelial dysfunction. ${ }^{8}$ Treatment with the antioxidant vitamin C produced a significant inhibition of oxidative stress, an improvement in endothelial function, and a reduction of cardiac hypertrophy. ${ }^{8}$ Another recent study by MacCarthy and colleagues ${ }^{9}$ using the same model describes an increase in myocardial $\mathrm{NAD}(\mathrm{P}) \mathrm{H}$ oxidase activity accompanied by a defect in cardiac contractile function. Application of exogenous vitamin $\mathrm{C}$ to the isolated heart preparation reversed this defect.

\section{A ROLE FOR NITRIC OXIDE?}

Clearly these studies suggest that there is a link between AII, oxidative stress, and cardiac function. Given the well established fact that NO exerts significant and biologically relevant effects on cardiac function, in particular selectively enhancing myocardial relaxation, ${ }^{10}$ it seems likely that endothelium derived NO might provide this link. Indeed, decreased NO bioavailability in the face of an oxidising environment is the probable cause of the observed defect in cardiac function in this model.

The importance of NO in the heart must not be underestimated since it has many effects on the myocardium other than its role as an antioxidant. For instance, its antiproliferative effects are exemplified by the demonstration that chronic inhibition of NO synthesis leads to cardiac hypertrophy independently of effects on systemic blood pressure. ${ }^{11}$ In addition, NO has been shown to inhibit both angiotensin converting enzyme (ACE) and matrix metalloproteinase activity, which contributes directly to the myocardial remodelling process in the transition from compensatory hypertrophy to cardiac failure. ${ }^{12}$

It is also interesting that physiologically relevant concentrations of $\mathrm{NO}$ can have a controlling influence on mitochondrial respiration. Whether the controlling effect of NO is faulty or absent in FRDA is not known, however. It has been suggested that in the absence of $\mathrm{NO}$, mitochondrial dysfunction can lead to cardiac myocyte apoptosis and necrosis. Inhibition of the oxidative stress induced decrease in NO bioavailability would therefore seem to be a desirable therapeutic strategy, though its use in FRDA needs further investigation.

The benefit of vitamin $\mathrm{C}$ in cardiac hypertrophy would seem, at least in part, to rely on an increase in NO bioavailability, but whether this mechanism is responsible for the beneficial effects of idebenone in FRDA is not known. There are no reports in the literature documenting endothelial function in FRDA patients, an area clearly waiting to be explored

\section{NOVEL ANTIOXIDANT TREATMENT?}

Are other antioxidant therapeutic options available for the treatment of cardiac hypertrophy? Of interest is the demonstration that the 3-hydroxy-3-methylglutaryl-CoA reductase inhibitor simvastatin has been shown to inhibit cardiac hypertrophy and ACE activity, and improve left ventricular performance in rats with aortic stenosis in a cholesterol independent manner. ${ }^{13}$ More recently, it has been suggested that simvastatin exerts its effect on cardiac hypertrophy via an antioxidant mechanism involving inhibition of $\mathrm{Racl},{ }^{14}$ the small G protein involved in AII induced ROS production. Furthermore, the statins have also been shown to restore endothelial function and thus NO bioavailability, possibly via the upregulation of endothelial NO synthase. The role of the statins in the treatment of cardiac hypertrophy has yet to be established, however.

A further intervention that has also been shown to attenuate the development of hypertrophy in a pressure overload murine model is $17 \beta$-oestradiol, though the mechanism remains obscure. ${ }^{15}$ That left ventricular hypertrophy displays significant sex differences is well known, ${ }^{16}$ with women displaying a distinct survival advantage when it is present. Given that $17 \beta$-oestradiol is the predominant oestrogen in premenopausal women and has major cardiovascular effects, including a regulatory role in the expression of cardiac endothelial and inducible NO synthase, its use in the treatment of postmenopausal women with cardiac hypertrophy should possibly be considered.

There are without doubt many factors other than oxidative stress that contribute to the pathology of cardiac hypertrophy. Nevertheless oxidative stress and its involvement in this common cardiac condition is becoming more of a reality than previously thought, requiring not such a great leap of faith after all!

\section{REFERENCES}

1 Hausse A, Aggoun Y, Bonnet D, et al. Idebenone and reduced cardiac hypertrophy in Friedreich's ataxia. Heart 2002;87:346-9.

2 Gabsi S, Gouider-Khouja N, Belal S, et al. Effect of vitamin E supplementation in patients with ataxia with vitamin $\mathrm{E}$ deficiency. Eur J Neurol 2001;8:477-81

3 Ide T, Tsutsui $\mathrm{H}$, Kinugawa $\mathrm{S}$, et al. Mitochondrial electron transport complex I is a potential source of oxygen free radicals in the failing myocardium. Circ Res 1999:85:357-63.

4 Chantrel-Groussard K, Geromel V, Puccio H, et al. Disabled early recruitment of antioxidant defenses in Friedreich's ataxia. Hum Mol Genet $2001 ; 10: 2061-7$

5 Lodi R, Rajagopalan B, Blamire AM, et al. Cardiac energetics are abnormal in Friedreich ataxia patients in the absence of cardiac dysfunction and hypertrophy: an in vivo $31 \mathrm{P}$ magnetic resonance spectroscopy study. Cardiovasc Res 2001;52:111-9.

6 Wang Y. Signal transduction in cardiac hypertrophy - dissecting compensatory versus pathological pathways utilizing a transgenic approach. Curr Opin Pharmacol 2001:1:134-40.

7 Lang D, Mosfer SI, Shakesby A, et al. Coronary microvascular endothelial cell redox state in left ventricular hypertrophy: the role of angiotensin II. Circ Res 2000;86:463-9.

8 Bell JP, Mosfer SI, Lang D, et al. Vitamin C and quinapril abrogate LVH and endothelial dysfunction in aortic-banded guinea pigs. Am J Physiol Heart Circ Physiol 2001;281:H1704-10.

9 MacCarthy PA, Grieve DJ, Li JM, et al. Impaired endothelial regulation of ventricular relaxation in cardiac hypertrophy: role of reactive oxygen species and NADPH oxidase. Circulation 2001;104:2967-74.

10 Shah AM, MacCarthy PA. Paracrine and autocrine effects of nitric oxide on myocardial function. Pharmacol Ther 2000;86:49-86.

11 Sanada S, Kitakaze M, Node K, et al. Differential subcellular actions of ACE inhibitors and $A T(1)$ receptor antagonists on cardiac remodeling induced by chronic inhibition of $\mathrm{NO}$ synthesis in rats. Hypertension $2001 \cdot 38 \cdot 404-11$

12 Peterson JT, Hallak H, Johnson L, et al. Matrix metalloproteinase inhibition attenuates left ventricular remodeling and dysfunction in a rat model of progressive heart failure. Circulation 2001;103:2303-9.

13 Luo JD, Zhang WW, Zhang GP, et al. Simvastatin inhibits cardiac hypertrophy and angiotensin-converting enzyme activity in rats with aortic stenosis. Clin Exp Pharmacol Physiol 1999;26:903-8.

14 Takemoto $M$, Node K, Nakagami H, et al. Statins as antioxidant therapy for preventing cardiac myocyte hypertrophy. J Clin Invest 2001; 108:1429-37.

15 van Eickels $M$, Grohe C, Cleutjens JP, et al. 17beta-estradiol attenuates the development of pressure-overload hypertrophy. Circulation $2001 ; 104: 1419-23$

16 Hayward CS, Kelly RP, Collins P. The roles of gender, the menopause and hormone replacement on cardiovascular function. Cardiovasc Res 2000:46:28-49. 